\title{
Anmerkungen zum Salienzbegriff in der Soziolinguistik
}

Peter Auer (Freiburg i. Br.)

\begin{abstract}
In this paper I argue that the psychological notion of salience should be kept strictly apart from its causes and effects (on language change or language accommodation). I further argue that we should distinguish between physiological, cognitive and sociolinguistic factors contributing to salience. Finally, it is argued that salience based on social stereotyping is the best predictor of change and accommodation, but needs to be supported by the right evaluation of the salient feature in order to have an effect.
\end{abstract}

\section{Salienz - ein problematischer Begriff}

Der Begriff der Salienz hat in der (germanistischen) Soziolinguistik derzeit wieder Konjunktur. Dies ist möglicherweise ein Seiteneffekt des Interesses an der Wahrnehmung von sprachlicher Variation, die immer mehr zur Untersuchung der Variation selbst hinzutritt (perceptual dialectology etc.; cf. etwa Anders/Hundt/Lasch 2010). Salienz ist ja ein durch und durch auf die Wahrnehmung bezogener Begriff, der den Hörer/die Hörerin in den Mittelpunkt stellt. Die Salienz eines sprachlichen Merkmals kann also nicht in ihm selbst liegen, sondern nur in der Art und Weise, wie es gehört wird. Allerdings ist diese Art der Wahrnehmung nicht idiosynkratisch, sondern sie wird in der Regel von vielen Menschen geteilt. Es stellt sich deshalb die Frage, wie ein sprachliches Merkmal für eine Gruppe von Sprechern salient werden kann (Gründe für Salienz). Für die Soziolinguistik gilt überdies, dass Salienz fast nie als Selbstzweck untersucht wird; vielmehr richtet sich schon seit Schirmunskis Forschungen zur Auffälligkeit von Dialektmerkmalen das Interesse auf Fragen des Sprachwandels (insbesondere der sprachlichen Konvergenz) und der sprachlichen Akkommodation. Die Vermutung ist, dass saliente Merkmale sich dabei anders verhalten als nicht-saliente Merkmale (etwa zuerst erworben oder zuerst abgebaut werden). Hier geht es also um Auswirkungen von Salienz.

Die (sozio-)linguistische Diskussion des Begriffs ist allerdings oft von einer Vermischung der Salienz selbst (i. S. v. Auffälligkeit) mit ihren Ursachen und Wirkungen gekennzeichnet; das macht die Verständigung darüber, was Salienz ist, oft schwierig. ${ }^{1}$ Insbesondere werden oft Ursachen für Salienz als unabhängige Variablen direkt mit Sprachwandel als abhängiger Variable in Verbindung gebracht - etwa wenn der beobachtete Sprachwandel durch die Salienz des Merkmals erklärt wird, diese aber mit Faktoren wie Phonemstatus, geografischer Reichweite oder Frequenz (bzw. Seltenheit) gleichgesetzt oder durch sie operationalisiert wird. Dieses Vorgehen klammert die Untersuchung der Salienz selbst aus; sie kommt als perzeptorisches Phänomen gar nicht mehr vor:

\footnotetext{
${ }^{1}$ Diese Kritik schließt übrigens die Diskussion in Auer, Barden und Großkopf 1996 und Auer 2001 mit ein. Linguistik online 66, 4/14 - http://dx.doi.org/10.13092/lo.66.1569 


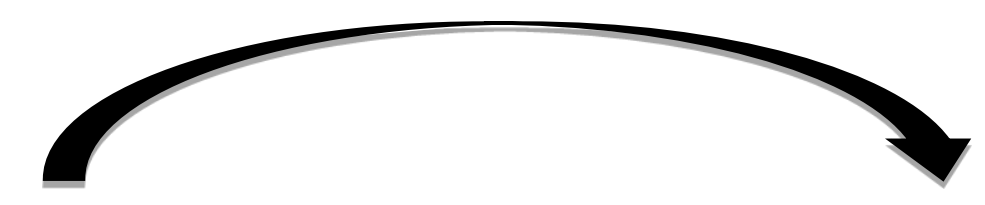

URSACHE VON SALIENZ

z. B.

Phonemstatus

Frequenz

geografische Reichweite

Seltenheit

etc...
WIRKUNG VON SALIENZ

Z. B.

( SALIENZ) Akkommodation

Sprachwandel

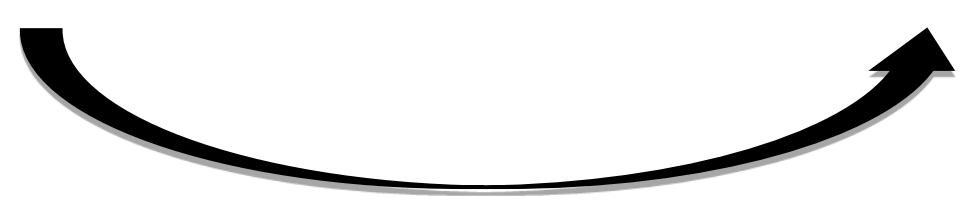

Abbildung 1: Ursachen und Wirkungen von Salienz kurzgeschlossen.

Im Folgenden möchte ich im Interesse der begrifflichen und theoretischen Klärung dafür plädieren, sorgfältiger zwischen Ursachen und Wirkungen von Salienz einerseits und der Definition bzw. Operationalisierung von Salienz selbst andererseits zu unterscheiden. So stellen sich drei ganz unterschiedliche Fragen:

(1) Die Frage nach den Kriterien für Salienz: Wie lässt sich ein salientes Merkmal erkennen? Wie lässt es sich von einem weniger salienten Merkmal unterscheiden? Wie lässt sich Salienz operationalisieren? Wie lässt sie sich gegebenenfalls messen?

(2) Die Frage nach den Ursachen von Salienz: Gibt es bestimmte Eigenschaften von Merkmalen oder deren Kontexten, die Salienz bedingen, also ein sprachliches Merkmal salient machen?

(3) Die Frage nach den Auswirkungen von Salienz, besonders auf den Sprachwandel: Werden saliente Merkmale im Varietätenkontakt in der akkommodierenden Varietät besonders schnell abgebaut? Werden salientere Merkmale der Zielvarietät schneller erworben als weniger saliente?

Es ist offensichtlich, dass eine Vermischung von (1) und (3) zur Zirkularität führt, denn dann wird Salienz sowohl als erklärender Faktor für Sprachwandel als auch Sprachwandel als Kriterium für Salienz verwendet. Diese Kritik trifft zwar nicht auf Schirmunski (1928/1929 und 1930) zu, obwohl es ihm mehrfach vorgeworfen worden ist (cf. Trost 1968), wohl aber z. B. auf Trudgill (1986: 11): Eines seiner Kriterien für Salienz ist in der Tat Sprachwandel. Will man eine terminologische Unterscheidung zwischen der Salienz eines Merkmals und seiner empirisch nachweisbaren Abbauwahrscheinlichkeit im Sprachwandel bei Dialektkontakt oder Standard/Dialekt-Kontakt treffen, so bietet sich für letztere die Unterscheidung zwischen primären und sekundären (bzw. im Bedarfsfall tertiären etc.) Merkmalen an: Primäre Merkmale einer Varietät könnte man definieren als diejenigen, die im Wandel zuerst aufgegeben werden etc. (cf. Lenz 2010). Dies entspricht zwar nicht der Definition der primären und sekundären 
Merkmale bei Schirmunski (der saliente und primäre Merkmale gleichsetzt), erleichtert aber die konzeptuelle Trennung von Salienz und ihren Auswirkungen.

Die Vermischung von(1) und (2) ist noch viel häufiger, vielleicht allerdings auch verzeihlicher. Tatsächlich lassen sich nämlich Kriterien und Ursachen in manchen Fällen nicht scharf trennen. Ein Beispiel soll dies zeigen. Ein valider indirekter Beleg für die Salienz von sprachlichen Merkmalen sind Selbst- und Fremdkorrekturen. Im folgenden Beispiel handelt es sich um eine Fremdkorrektur, mittels derer ein jugendlicher Sprecher mit türkischem Migrationshintergrund in Stuttgart seinen Freund mit kosovo-albanischem Sprachhintergrund wegen eines bestimmten formalen Merkmals seiner Sprache korrigiert:

(1) (BC_JA_01, 426-432)

(IL ist ein 15-jähriger Kosovo-Albaner, MV sein 15-jähriger türkischer Freund; es geht um die Schulprojekte der Jugendlichen, nach denen JS, die Interviewerin EX, gefragt hat)

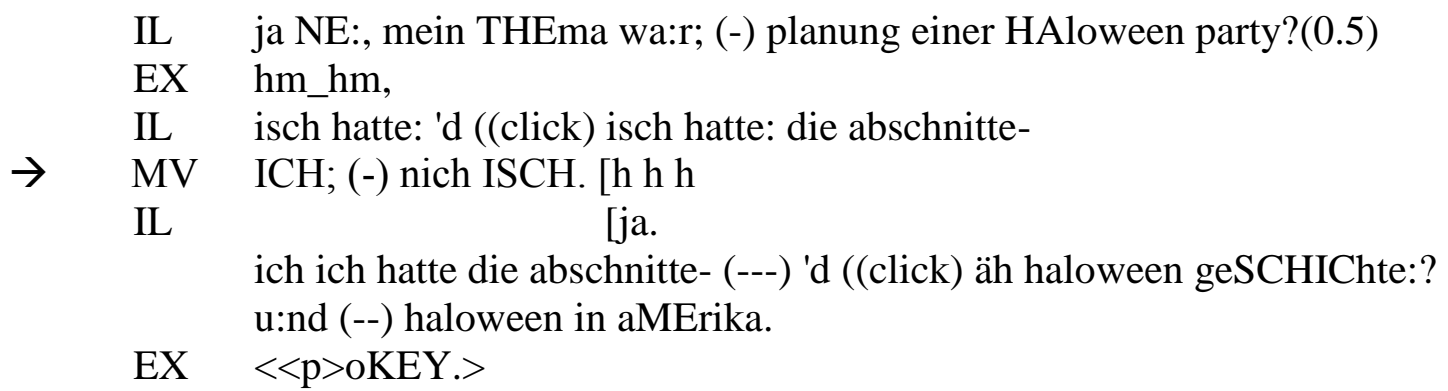

Die Tatsache, dass MV die Koronalisierung von ich in der Äußerung IL's explizit tadelt, worauf IL seine Äußerung korrigiert und mit dem "richtigen", standardsprachlichen Frikativ fortsetzt, ist ein starkes Indiz dafür, dass das Merkmal in dieser Gruppe von Jugendlichen wahrgenommen wird, also salient ist. Das Vorkommen von Reparaturen kann also als Kriterium für Salienz verwendet werden. Zugleich machen solche Reparaturen Merkmale aber auch salient, d. h. sie sind eines der Verfahren, durch die ein Merkmal in einer Gruppe von Sprechern auffällig wird (cf. dazu unten mehr).

\section{$2 \quad$ Was ist salient?}

Die Salienz eines Merkmals lässt sich nur vor einem Hintergrund erkennen, aus dem es mehr oder weniger stark heraussticht (wie die Etymologie $>$ 1t. salire 'hervorspringen' nahelegt). Salienz ist also ein typisches Figur/Grund-Problem. Als solches gehört es in den Bereich der Wahrnehmung. Es ist sinnvoll, drei Bedingungsgefüge für Salienz zu unterscheiden, die das fragliche sprachliche Merkmal als Figur vor einem je unterschiedlichen Grund profilieren.

(a) Physiologisch bedingte Salienz: Solang der Grund, vor dem das fragliche Merkmal sich als Figur abhebt, wie dieses sensorisch zugänglich ist und die Beziehung zwischen beiden ausschließlich durch die Physiologie der Wahrnehmung bedingt ist, lässt sich von perzeptorischer Salienz im engeren Sinn (oder physiologischer Salienz) sprechen. (Ellis i. Dr. verwendet den Begriff "physikalische Salienz", der allerdings die Auffälligkeit zu sehr in das wahrgenommene Objekt verlagert.) Selbst beim Hören einer unbekannten Sprache wird zum Beispiel die Aufmerksamkeit auf bestimmte lautliche Elemente gelenkt, die von ihrer Umgebung besonders gut gesondert werden können, zum Beispiel weil sie lauter oder langsamer gesprochen werden.

(b) Kognitiv bedingte Salienz: In der Regel wird ein sprachlicher Stimulus aber vor dem Hintergrund des gesamten sprachlichen Wissens perzipiert, über das der Wahrnehmende verfügt. Normalerweise ist das ein Repertoire an sprachlichen Formen, von denen einige aktiv, viele andere passiv beherrscht werden. Das eigene Repertoire von Formen ist also der Grund, 
von dem sich das gehörte sprachliche Ereignis als 'Figur' abhebt. Dies ist umso mehr der Fall, je unbekannter und daher unerwarteter das Merkmal für den Wahrnehmenden ist (cf. Rázs 2013). Kognitiv bedingte Salienz (und auch soziolinguistisch bedingte, siehe unten) unterscheidet sich daher ganz wesentlich von der physiologischen. Sie beruht immer auf einem Gegensatz zwischen verschiedenen Sprechweisen (cf. Lenz 2010): Merkmale einer Sprechweise fallen erst im Vergleich zu einer anderen auf, z. B. die eines Dialekts im mit dem Standard.

Allerdings ist die für die Salienz verantwortliche Differenz zwischen Gehörtem und Gewusstem (und daher Erwartetem) nicht kategorisch; wir sind mit anderen Sprechweisen (anderen Dialekten, etc.) mehr oder weniger vertraut. Ein Beispiel mag dies klar machen. Der Unterschied zwischen ihrem eigenen /oi/ und mittelbair. /oa/ (für mhd. ei, wie etwa in ich weiß) wird von Sprechern des Zentralschwäbischen sicherlich wahrgenommen, er ist jedoch Teil des passiven Wissens über das Bairische und daher nicht überraschend. /oa/ entspricht dem gängigen Klischee des (am Münchner Dialekt orientierten) Mittelbairischen, und dieses Klischee ist für viele Schwaben auch durch persönliche Erfahrungen unterfüttert. Verwendet ein Sprecher jedoch nordbair. /ou/ anstelle von allgemein-schwäb. /ue/ (für mhd. uo, wie etwa in $B u b$ ), hebt sich diese Form nicht nur deutlich vom eigenen schwäbischen Dialekt (und dem Standarddeutschen) $a b$, sondern sie wird für die meisten Schwaben überdies auch innerhalb ihres passiven Wissens über das Bairische unerwartet sein. /bou/ entspricht weder der bekannten mittelbair. Form (die wäre/bua/), noch wird die Form von vielen Sprechern verwendet; entsprechend gering ist die Wahrscheinlichkeit, dass sie Teil des passiven Repertoires schwäbischer Sprecher ist, und entsprechend auffällig ist eine solche Aussprache.

(c) Soziolinguistisch bedingte Salienz ist jedoch auch auf diese Weise noch nicht angemessen erfasst. Sie geht über das auffällig Andere hinaus und darf deshalb nicht mit dem Unterschied zwischen Erwartetem-Gewusstem und Unerwartetem-Überraschendem gleichgesetzt werden. Es ist ein Gemeinplatz der Psychologie der Wahrnehmung, dass die Auffälligkeit eines Stimulus auch von der Motivation des wahrnehmenden Subjekts abhängig ist und daher emotionale und motivationale Komponenten hat. Ellis (i. Dr.) spricht hier von psychologischer Salienz. So sticht ein emotional besonders besetzter Stimulus besonders aus seiner Umgebung heraus, ebenso wie ein Objekt, das die augenblicklichen Bedürfnisse des Wahrnehmenden, wie Hunger oder Durst, befriedigen kann, mehr ins Auge fällt als eines, das dies nicht tut.

In der Soziolinguistik entspricht dieser Dimension von Salienz die soziale und affektive Bewertung des Merkmals, die daraus resultiert, dass dieses als Index für einen bestimmten sozialen Sprechertyp fungiert, der mit denselben Bewertungen verbunden ist. Bei den kognitiv salienten Merkmalen ist der Grund, vor dem sie sich als Figur profilieren, das (mehr oder weniger) Bekannte. Soziolinguistisch saliente Merkmale beziehen hingegen ihre Salienz aus der Stärke der negativen oder positiven sozialen Bewertung des Merkmals auf einem relevanten sprachideologischen Hintergrund (cf. Auer 2013).

Nicht alle kognitiv salienten Merkmale sind also auch soziolinguistisch salient. Zum Beispiel kann man unter Dialektsprechern relativ leicht Aufzählungen bewusster, kognitiv salienter Merkmale der Nachbardialekte elizitieren. So listet die folgende Tabelle die am häufigsten genannten Merkmale der alemannischen Nachbardialekte in einem relativ kleinräumigen Erhebungsgebiet im südlichen Rheintal auf, geordnet nach der Anzahl der Sprecher, die dieses Merkmal genannt haben (letzte Spalte) (Grundlage: 218 Sprecher, aus: Stöckle 2013: 514). 


\begin{tabular}{|c|c|c|c|c|}
\hline Phänomen & Kategorie & Beispiele & $\begin{array}{l}\text { \# Merk- } \\
\text { male }\end{array}$ & $\begin{array}{l}\text { \# verschiede- } \\
\text { ne Sprecher }\end{array}$ \\
\hline $\begin{array}{l}k \text {-Verschiebung bzw. } \\
i c h / a c h \text {-Alternation }\end{array}$ & Konsonantismus & "mehr $[\mathrm{x}] "$; Kind $>[\mathrm{xmt}] ;$ komm $>[\mathrm{x} \mho \mathrm{m}]$ & 181 & $113(51,8 \%)$ \\
\hline $\begin{array}{l}\text { standardnähere Vari- } \\
\text { ante }\end{array}$ & Phonologie; Lexik & bei uns $>$ [bi ons] (statt [bi y:s]) & 128 & $73(33,5 \%)$ \\
\hline Dialektstärke & $\begin{array}{l}\text { metasprachliche } \\
\text { Charakterisierung }\end{array}$ & $\begin{array}{l}\text { starker Dialekt: „sehr viel breiter", „extremer"; } \\
\text { schwacher Dialekt: „nicht das urige Aleman- } \\
\text { nisch", „nicht so breit" }\end{array}$ & 105 & $64(29,4 \%)$ \\
\hline Senkung von $e$ zu $a$ & Vokalismus & Nebel $>$ [na:bl]; Messer [mase] & 67 & $47(21,6 \%)$ \\
\hline$a$-Verdumpfung & Vokalismus & $\begin{array}{l}\text { Mantel, Kandel > [mondl kondl]; "o anstatt } \\
\text { a" }\end{array}$ & 69 & $47(21,6 \%)$ \\
\hline Entrundung & Vokalismus & $\begin{array}{l}\text { Büchse }>\text { [biks]; Häuschen }>\text { [hi:sli] (statt } \\
\text { [hy:slr]) }\end{array}$ & 57 & $39(17,9 \%)$ \\
\hline Richtungsadverben & $\begin{array}{l}\text { Phonologie; Lexik; } \\
\text { Morphologie }\end{array}$ & hinauf $>$ [ชfə], [u:ə]; hinab > [a:bə], [nab] & 78 & $34(15,6 \%)$ \\
\hline gerundete Vokale & Vokalismus & $\begin{array}{l}\text { Apfel }>\text { [œpfl] (statt [عpfl]); Häuser > [hy:se] } \\
\text { (statt [hi:se]) }\end{array}$ & 38 & $30(13,8 \%)$ \\
\hline$u$-Palatalisierung & Vokalismus & $\begin{array}{l}\text { Nudeln }>\text { [ny:dle]; Bauch }>\text { [by:x] (statt } \\
\text { [bu:x]); „mehr das ü“ }\end{array}$ & 42 & $29(13,3 \%)$ \\
\hline au-Hebung & Vokalismus & $\begin{array}{l}\text { blau }>[\mathrm{blol}] ; \text { Frau }>[\mathrm{froI}] ; \text {, } \text { machen aus } a u \\
\text { ein } e u^{6}\end{array}$ & 34 & $26(11,9 \%)$ \\
\hline $\begin{array}{l}\text { Interferenz (Franzö- } \\
\text { sisch) }\end{array}$ & $\begin{array}{l}\text { Phonologie; Lexik; } \\
\text { metasprachliche } \\
\text { Charakterisierung }\end{array}$ & $\begin{array}{l}\text { Spülbecken > „Lavabo"; "Mischmasch zwi- } \\
\text { schen Französisch und Alemannisch"; „ins } \\
\text { Französisch gehende Laute" }\end{array}$ & 40 & $26(11,9 \%)$ \\
\hline „sagen" & Vokalismus & sagen > [se:gə] & 32 & $23(10,6 \%)$ \\
\hline „Kartoffel" & Lexik & $\begin{array}{l}\text { „Herdapfel" [heedœpfl]; „Grundbirne" [grom- } \\
\text { br:Re] }\end{array}$ & 38 & $22(10,1 \%)$ \\
\hline
\end{tabular}

Tabelle 1: Äm häufigsten genannte Merkmale der Nachbardialekte in einem südwestdeutschen Erhebungsgebiet (zwischen Schweizer Grenze und Linie Elzach/Herbolzheim im Norden, sowie zwischen französischer Grenze und Linie Elzach/Tiengen im Osten), geordnet nach der Anzahl der Sprecher, die dieses Merkmal genannt haben (letzte Spalte) (Grundlage: 218 Sprecher, aus: Stöckle 2013: 514).

Unabhängig vom Grad ihrer kognitiven Salienz werden Merkmale wie der Umlaut von sagen, die Palatalisierung von /u:/ oder die Senkung von /e/ aber zumindest bei isolierter Nennung nicht sozial und affektiv bewertet. Sie werden zwar unterschiedlichen Gebieten im Untersuchungsraum zugewiesen, erfahren aber keine nennenswerte soziale Interpretation. Ganz anders verhält es sich zum Beispiel mit der schon erwähnten Koronalisierung des palatalen Frikativs (s. Bsp. (1)). Sie ist hoch stigmatisiert und mit stark affektiv besetzten, sozialen Kategorisierungen verbunden. Dies belegen mediale Bezugnahmen auf das Merkmal wie Bsp. (2), ein Ausschnitt aus der berühmten SPIEGEL-Titelgeschichte über die Rütli-Schule in Neukölln, und - in der Umkehrung - im Comic in Abb. (2):

(2) (SPIEGEL 14, 2006, Titelgeschichte "Die verlorene Welt", Fettung P.A.) Aufklärung? Bildung? Lernen, für Zensuren, vielleicht sogar fürs Leben? Was soll der Scheiß?

So reden die Bewohner dieser Welt. Ey, Mann, ey. Nutte. Killer. Krass. Es gibt viele "sch"- und "ch"-Laute in dieser Sprache, kaum noch ganze Sätze. Dreckische Deutsche, so reden sie. 


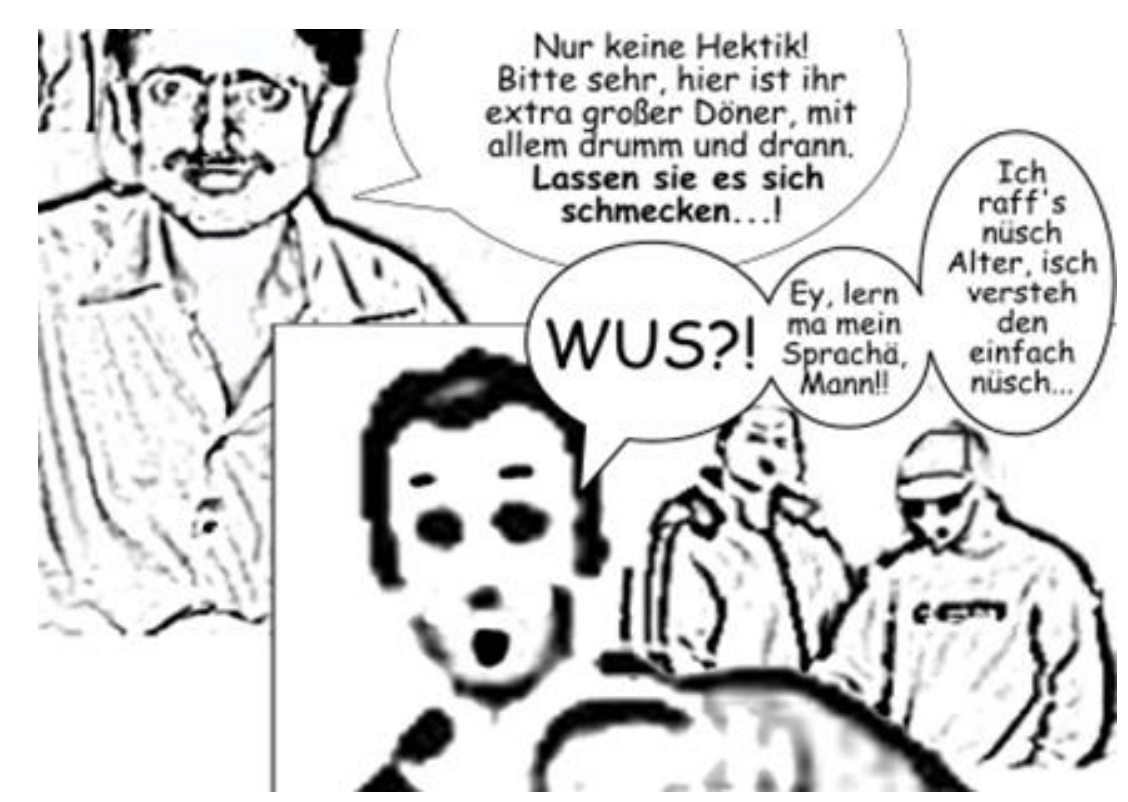

Abbildung 2: Ausschnitt aus einem Comic; gezeigt wird die Interaktion zwischen dem türkischen DönerVerkäufer, der lupenreines Hochdeutsch spricht, und einer Gruppe deutscher Großstadtjugendlicher, die ihre eigene Sprache an das Stereotyp der türkischen "Kanaksprak" angepasst haben. Vgl. die Koronalisierung in nüsch, isch (http://1.bp.blogspot.com/_0msJlvGVVi8/TOrinlLivRI/AAAAAAAAADY/ vsEYPaJnNfM/s1600/comic_doener.jpg).

Soziolinguistisch saliente Merkmale sind also solche, die eine sozial-affektive Bewertung erfahren. Wichtig ist, dass das Merkmal allein bedeutungsvoll ist. Oft ist es für die Mitglieder einer Sprechgemeinschaft gar nicht relevant, einzelne Merkmale zu isolieren und mit sozialer Bedeutung zu verbinden. Vielmehr erkennen sie einen Stil (cf. Eckert 2004), der aus einer großen Zahl kookkurrierender Merkmale besteht und holistisch interpretiert wird. Soziolinguistisch saliente Einzelmerkmale sind also eher die Ausnahme als die Regel. Wenn aber eine soziale Bewertung des Merkmals vorliegt, so die These dieses Beitrags, ist das Merkmal auffälliger als eines, das lediglich kognitiv oder gar nur physiologisch als Figur vor einem Grund profiliert ist.

\section{$3 \quad$ Kriterien für Salienz}

Da Salienz ein Phänomen der Wahrnehmung ist, muss ihre Untersuchung in erster Linie mit experimentellen Verfahren erfolgen, die die Auffälligkeit des Merkmals in einem bestimmten Kontext messen. Nur ein kleiner Teil salienter Merkmale - sozusagen die Spitze des Eisbergs - lassen sich durch kontextfreie Abfragen wie in Tab. (1) elizitieren oder aus dem expliziten Korrekturverhalten in spontanen Interaktionen wie in Bsp. (1) belegen. Die Wahl der Stimuli in experimentellen Untersuchungen zur Salienz von sprachlichen Merkmalen ist von den Faktoren abhängig, die sie bedingt. Um physiologisch bedingte Salienz zu untersuchen, ist es sinnvoll, Tests zu konzipieren, bei denen den Probanden die Sprache der Stimuli nicht bekannt ist. Für die hier vor allem interessierende kognitiv und soziolinguistisch bedingte Salienz sind in letzter Zeit in verschiedenen Projekten experimentelle und quasi-experimentelle Verfahren entwickelt und erprobt worden (vgl. etwa im SIN-Projekt, siehe Elmentaler/Gessinger/Wirrer 2010). Im einfachsten Fall müssen die Probanden die fraglichen Merkmale im gesprochenen oder geschriebenen Stimulusmaterial identifizieren; die Anzahl der erkannten Beispiele wird als abhängige Variable berechnet. Feiner differenzierende Verfahren können mit Reaktionszeitmessungen arbeiten. Die Analyse der soziolinguistischen Salienz erfordert die Verwendung von Stimuli, die unterschiedlicher sozialer Bewertung unterliegen und auf der emotionalen Dimension mehr oder weniger starke Reaktionen hervorrufen. 


\section{$4 \quad$ Ursachen für Salienz}

Die Unterscheidung zwischen physiologisch, kognitiv und soziolinguistisch bedingter Salienz kann nun weiter differenziert werden.

\subsection{Physiologische Ursachen}

In der mündlichen Sprache werden solche Lautereignisse besonders gut wahrgenommen, die durch prosodische Parameter wie längere Dauer, stärkere Grundfrequenzbewegung und höhere Intensität (Lautstärke) hervorgehoben werden. Entsprechend sind in einer Akzentsprache die lautlichen Ereignisse in der Akzentposition ceteris paribus salienter als die in den unakzentuierten (oder nebenakzentuierten) Silben. Ein anderer Faktor, der Salienz auf dieser einfachsten Ebene der Wahrnehmung begründen kann, ist die Position eines sprachlichen Ereignisses am Anfang bzw. am Ende einer Einheit (z. B. der Äußerung, aber auch der Silbe). Es ist bekannt, dass neben der Akzentposition auch die End- und Anfangsposition im kindlichen Erstspracherwerb eine wichtige Rolle spielen, eben weil sie perzeptiv besonders gut zugänglich sind. Des Weiteren sind sprachliche Ereignisse vermutlich besser wahrnehmbar und daher auch potentiell salienter, die sich innerhalb eines kleinen Zeitfensters mehrfach wiederholen. Überdies spielen intrinsische Merkmale von einzelnen Lautereignissen (etwa die Anzahl und Größe der beteiligten artikulatorischen Gesten) eine Rolle. Graduierliche und variable phonetische Prozesse sind schlechter wahrnehmbar als dichotomische und kategorische.

\subsection{Kognitive Ursachen}

Kognitiv bedingte Salienz ist davon abhängig, wie stark sich ein wahrgenommenes sprachliches Merkmal als Figur vor dem Grund des sprachlichen Repertoires des Wahrnehmenden abhebt. Daraus ergeben sich die folgenden Prädiktoren für Salienz:

- was im wahrgenommenen Sprachmaterial mit der eigenen Sprache übereinstimmt, ist am wenigsten salient;

- was sich im wahrgenommenen Sprachmaterial zwar von der eigenen Sprache unterscheidet, aber aufgrund des Wissens darüber, wie bestimmte Typen von Menschen sprechen, erwartbar ist, ist von mittlerer kognitiver Salienz;

- was sich im wahrgenommenen Sprachmaterial von der eigenen Sprache unterscheidet und überdies der Erwartung über das typische Verhalten des Sprechers oder der Sprecherin widerspricht, ist stark salient;

- was sich im wahrgenommenen Sprachmaterial von der eigenen Sprache unterscheidet und unbekannt ist, ist ebenfalls stark salient (weil völlig unerwartet), allerdings nur unter der Voraussetzung, dass das Merkmal physiologisch überhaupt bemerkt wird.

Auf dem Hintergrund dieser Prädiktoren lässt sich leicht erklären, warum Salienz und areale Verbreitung ("Reichweite") eines Merkmals korrelieren. Stärker verbreitete Merkmale sind vielen Menschen bewusst und für sie relativ erwartbar, areal stark eingeschränkte Merkmale nicht. Die geografische Verbreitung ist aber keine direkte Ursache für Salienz, sondern lediglich indirekt damit verbunden.

Allerdings ist es nicht ausreichend, kognitiv bedingte Salienz ausschließlich in Abhängigkeit vom sprachlichen Wissen der wahrnehmenden Personen zu sehen. Auch die Stellung des fraglichen Merkmals im Sprachsystem spielt eine Rolle: Die Salienz eines sprachlichen Merkmals ist umso größer, je mehr das Merkmal in der jeweiligen sprachlichen Varietät funktional belegt ist. Das gilt bereits für intralektale Salienz: Sprecher einer Varietät sind sich eher solcher sprachlicher Merkmale ihrer eigenen Sprache bewusst, die Bedeutung haben (daher eher Inhalts- als Formwörter, eher Wörter als syntaktische Konstruktionen) oder 
zumindest Bedeutung unterscheiden (daher eher phonemische als subphonemische Merkmale) (cf. Silverstein 1981). Dasselbe gilt für die interlektale Salienz: Außerhalb von Bayern ist salient, wenn jemand für ein 'Brötchen' Semmel sagt (lexikalischer Kontrast), und Sprecher des Schweizerdeutschen finden das bundesdeutsche Müsli im Sinne von schweizerdt. Müesli ('cereals') sehr auffällig, weil es in ihrer eigenen Varietät ein Nagetier bezeichnet (phonemischer Kontrast). Hingegen sind manche syntaktischen und morphosyntaktischen Merkmale wenig salient, weil sie referenzsemantisch neutral sind. Beispiele sind der badische Akkusativ, also die Verwendung des morphologischen Nominativs für syntaktische Objekte wie in schöner Tag noch, oder die Verwendung von weil als Konjunktion mit Hauptsatzstellung statt als Subjunktion mit Nebensatzstellung - beide sind Gegenstand der expliziten Sprachkritik, werden aber in der eigenen Sprache wie auch der der anderen kaum wahrgenommen. Ebenso sind phonetische Merkmale weniger salient als phonemische. Der Grad an Aspiration der Plosive ist zum Beispiel zwar ein wesentliches phonetisches Merkmal, das nord- und süddeutsche Varietäten unterscheidet. Aber obwohl er durchaus zum holistischen Gesamteindruck von diesen Varietäten beiträgt, sind die Sprecher sich dieses Merkmals in der Regel weniger bewusst als eines phonemischen Kontrasts (etwa des Wechsels dat $\sim$ das, cf. unten, Bsp. (3)). Unter den phonetischen Merkmalen fallen wiederum diejenigen weniger auf, die nur einen geringen akustischen Kontrast bilden. So ist der Unterschied zwischen einem gerollten und einem frikativischen /r/-Laut phonetisch deutlich stärker als der zwischen einem hinteren (uvularen) und einem vorderen (apikalen) gerollten $/ \mathbf{r} /$. Entsprechend fällt der erste Kontrast mehr auf als der zweite.

\subsection{Soziale Ursachen}

Grundsätzlich sind zwar aus kognitiven Gründen saliente Merkmale eher dafür geeignet, auch sozial bewertet und daher noch salienter zu werden; allerdings ist der soziolinguistische Filter so stark, dass auch Merkmale, die aus verschiedenen kognitiven Gründen salient sein müssten, oft mangels sozialer Bewertung nur wenig auffallen (vgl. Tab.1), während umgekehrt Merkmale, die kognitiv gesehen wenig salient sein sollten, dennoch aufgrund ihrer sozialen Bewertung hoch auffällig sind. Tatsächlich sind viele subphonemische Merkmale sozial bewertet und daher hoch salient, wie etwa im Deutschen die verschiedenen r-Realisierungen.

Ein wichtiger, wenn auch nicht der einzige ${ }^{2}$ Weg, der sozialen Bewertung eines Merkmals führt, ist die Stereotypisierung. Wir drehen also die übliche Denkweise um und betrachten Stereotypisierung nicht als Folge der Salienz, sondern als ihre Ursache. ${ }^{3}$ Sprachliche Stereotypen stellen schemaartige Verbindungen zwischen einzelnen Varianten und sozialen Typen her und dienen auf diese Weise der Orientierung in der sozialen Welt: Sie schaffen soziale Ordnung in den Köpfen der Sprachbenutzer.

Stereotypisierte Merkmale werden leicht und schnell wahrgenommen. Stereotypen sind aber anders als Salienz kein Phänomen der Wahrnehmung, sondern des sprachlichen Wissens. Sie sind nicht auf die Wahrnehmung angewiesen und können auch ohne empirisches Korrelat tradiert werden. So ist es ein verbreitetes Stereotyp, dass alle Hamburger "über einen s-pitzen S-tein s-tolpern", obwohl dieses Merkmal faktisch nur sehr selten vorkommt (cf. Auer 1998/2010). Wenn es vorkommt, wird es zwar auch sofort wahrgenommen; das Stereotyp wird

\footnotetext{
${ }^{2}$ Vgl. die anhand von Bsp. (1) besprochene explizite Fremdreparatur. Im weiteren Sinn sind alle sprachbezogenen Sanktionierungen und (expliziten) Normierungen geeignet, Salienz herzustellen. Das schließt die Tätigkeit der Sprachnormierungsinstitutionen wie der Schulen mit ein.

3 Der Unterschied zwischen den beiden Sichtweisen lässt sich auf den Unterschied zwischen der älteren Stereotypen-Diskussion (maßgeblich beeinflusst von Labovs Unterscheidung zwischen Markern, Indikatoren und Stereotypen; cf. Labov 1972) und der neueren Diskussion von Indexikalität erster, zweiter und dritter Ordnung (insbesondere bei Johnstone, cf. etwa Johnstone/Andrus/Andrew 2006) abbilden.
} 
aber auch dann noch weiterleben, wenn der letzte Sprecher, der anlautend vor Obstruenten /s/ realisiert, verstorben ist.

Wie kommen nun sprachliche Stereotypen zustande? Bereits Schirmunski (1928/1929, 1930) nennt in diesem Zusammenhang den "Sprachspott", also Zuschreibungen wie die eben genannte ("alle Hamburger s-tolpern über den s-pitzen S-tein"). Zur Entstehung von Stereotypen tragen aber in erster Linie all jene Inszenierungen, Stilisierungen und Parodierungen von typisierten Sprechern bei, die im alltäglichen Gespräch wie auch in den Medien ständig produziert werden. In solchen sprachlichen Aktivitäten wird ein bestimmter Stil 'enkodiert' (enregisterment im Sinne von Agha 2003, 2007) und als Index mit einem sozialen Typus verbunden. Dabei können einzelne sprachliche Merkmale eine zentrale Rolle spielen.

Einige Beispiele für die Enkodierung von Stilen und der daraus resultierenden Stereotypisierung von sprachlichen Einzelmerkmalen wurden bereits oben besprochen (vgl. Bsp. (2) und Abb. (2)). Hier zwei weitere Beispiele, die sich auf Dialektmerkmale und deren soziale Bedeutung beziehen.

Das erste Beispiel ist einerseits medial, weil es aus einer deutschen reality-soap (Big Brother, 1. Staffel, aus dem Jahr 2000) stammt, andererseits interagieren die Beteiligten im Format eines informellen, konversationellen Austauschs. Die Rückbezüge zwischen Konversation und medialer Inszenierung werden in dem Ausschnitt selbst mehrfach thematisiert. Ich entnehme das Beispiel und seine Interpretation einem Beitrag von Birkner und Gilles (2008).

(2) (aus Birkner/Gilles 2008: 107-108)

((BB, 1. Staffel; Jürgen und Sabrina sitzen im Wohnzimmer des BB-Hauses, John geht draußen am Fenster vorbei. Sie spielen ein älteres Paar, das vor dem Fernseher sitzt und sich dabei langweilt, wie es die Übertragung aus dem BB-Haus sieht.))

$01 \quad$ Jrg: das wird praktisch morgen abend geZEIGT;

02 wie isch hier LIEge,

03 und er über_n HOF geht ne?

04 Sbr: hm,

$05 \quad$ ((kichert))

06 wir spielen jetzt öfters am tach (.) FERNsehn-

07 Jrg: ((kichert))

08 Sbr: <<all>intresSANT, ne Oder?

09 Jrg: ((lacht))

10 Sbr: (--) total. nä,>

$11 \quad$ ((kichert))

jetzt LIEGste da so schÖn;

(--) so (.) hier (.) am FERNseher so,

$\mathrm{ku}=\mathrm{mal}$;

15 Jrg: $\quad$ ((gähnt))

$\rightarrow \quad 17$ Sbr: $\quad<<$ ff, tief, rau>jib mal eben die FERNbedienung->

18 ((kichert))

19 Jrg: ((kichert))

20 Sbr: <<ff, hoch>NORBERT;

Jrg: ((lacht laut))

dat könn_ma doch net MA:Che;

dat is do der JÜ:Rgen;>

Sbr: $\quad$ < <ff, hoch>dat GEHT doch nIsch;

${ }^{\circ} \mathrm{h}$ das_s doch quAsi unser FREUND; $>$

26 Jrg: ((lacht) 


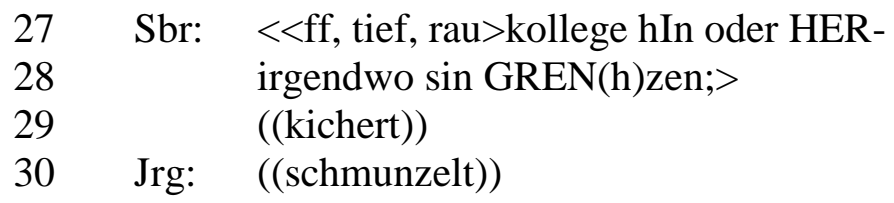

Jürgen und Sabrina inszenieren hier das fiktive Ehepaar "Norbert" und "Erna", die abends vor dem Fernseher sitzen (vgl. Z. 6, wir spielen jetzt öfters am tach (.) FERNsehn-). Zugleich sind sich die beiden der Tatsache bewusst, dass ihr eigenes triviales Leben im Big-Brother-Container ein mediales Ereignis ist und von anderen beobachtet wird (vgl. Z. 1-3, das wird praktisch morgen abend geZEIGT; wie isch hier LIEge, und er über_n HOF geht ne?). Diese Dopplung führt zur Inszenierung der Rezeption der eigenen Handlungen von Jürgen und Sabrina als Container-Bewohner durch die TV-Zuschauer "Norbert" und "Erna" in Z. 15-28. "Norbert" und "Erna" finden die BB-Übertragung über Jürgen und Sabrina langweilig, "Erna" verlangt daher nach der Fernbedienung, um sie wegzuschalten, überlegt sich aber dann, dass das für den BBBewohner Jürgen (also ihren tatsächlichen Gesprächspartner) ein Affront wäre.

Die Inszenierung der "Erna" wird sowohl prosodisch (Sabrina verändert ihre Stimmqualität) als auch durch den Wechsel in eine dialektnähere ripuarische Sprechweise indiziert. (Sowohl Sabrina als auch Jürgen kommen aus Köln, ihre unmarkierte Sprechweise wie in Z. 01-14 zeigt jedoch nur wenige segmentale Regionalismen.) Dieser Wechsel beruht auf einer kleinen Anzahl von sprachlichen Variablen (wie in Birkner/Gilles 2008 genauer beschrieben): dat für das, Dehnung von std. Kurzvokalen besonders in ma:che (Z. 21), nisch für std. nich(t), Apokope des $/ \mathrm{n} /$ in ma:che. Zugleich findet eine Enkodierung des sozialen Typus statt, der mit diesen Merkmalen assoziiert wird: der oder die traditionelle ältere Kölner/in im Arbeitermilieu, der/die die Abende vor der "Glotze" verbringt.

Das zweite Beispiel stammt aus einer schwäbischen Brauereiwerbungskampagne (SchwabenBräu-Werbung, um 2011, phonetische Zeichen und Klammern im Original, Beispiele aus verschiedenen Anzeigen):

(3) (a) ['heggschd per'se:nlich] (Bild von personalisierten Bierkrügen)

(b) [laofd 'nemme] (Bild eines alten VW-Käfers) - [laofd 'subbr] (Bier)

(c) [s'ällergre:schde] (Abbildung einer Bierflasche, weiterer Text: "so sprechen wir das schätzen wir")

(d) [sai 'dridde] (Bild der Ehefrau) - [sai 'zwoids] (Bier)

Solche Verschriftlichungen sind ein sehr starkes Mittel, um sprachliche Stereotypen zu erzeugen und zu tradieren. Im Beispiel werden als Merkmale des Stuttgarter Schwäbisch die Lenisierung der Plosive, die Entrundung, die Senkung von /i/ vor Nasal, bestimmte lexikalisierte Formen (nemme), der Umlaut in äller, die n-Apokope im Possessivum sein, die Realisierung von mhd. /ei/ als /oi/, die s-Palatalisierung, die Reduktion der schwa-haltigen Endsilben (zwoids) sowie die r-Erhaltung anstelle der r-Vokalisierung in Sequenzen aus Schwa und $/ \mathrm{r} /($ subbr) verwendet. Auch hier ist die Selbststilisierung mit einem bestimmten sozialen Typus verbunden, der in diesem Fall natürlich positiv bewertet wird: der Typus des bodenständigen, traditionsverbundenen Stuttgarters. Genauso wenig, wie Sabrina in Bsp. (3) alle sprachlichen Merkmale des Ripuarischen für ihre Stilisierung einsetzt, so werden auch in Dialektverschriftlichungen nicht alle Merkmale notiert - schon weil das Schriftsystem das oft gar nicht zulässt. (Auch wenn einige prosodische Zeichen für Dehnung und Akzent in (4) vermutlich zu Verfremdungszwecken - aus dem IPA entnommen sind, handelt es sich ja um keine phonetische Umschrift.) Dialektverschriftlichungen tendieren zudem zu graphemischen Hyperformen, die keiner lautlichen Differenz zum Standard entsprechen; vgl. etwa in (4)(c) das Dehnungszeichen oder in (4)(b) die Form $<$ ao $>$ für den Diphthong $a u$, beide im Schwäbischen nicht anders realisiert als im Standard. 
In beiden Beispielen beruht die (Selbst-)Stilisierung also auf der Verwendung eines bestimmten sozialen Stils, der neben einer selektiven Auswahl sprachlicher Merkmale auch non-verbale Verhaltensformen und Einstellungen, kurz: einen bestimmten Habitus umfasst. Nicht jedes der sprachlichen Merkmale, die zu diesem inszenierten Stil gehören, ist auch isoliert gesehen ein stereotypisiertes Merkmal. Um Stereotypen mit Einzelmerkmalen zu verbinden, müssen die Rezipienten quasi die Schnittmenge aus verschiedenen Stilisierungen bilden, die sie im Lauf ihrer sprachlichen Sozialisation zu hören oder lesen bekommen. Ein Merkmal muss also rekurrent vorkommen, damit es aus verschiedenen Stilen herausdestilliert werden kann und sozial bedeutungsvoll ist.

\section{Auswirkungen von Salienz auf Sprachwandel und sprachliche Akkommodation}

Die Frage der Salienz sprachlicher Merkmale ist seit Schirmunski eng an die Untersuchung von Sprachwandel geknüpft, insbesondere an die Frage der Konvergenz (und Divergenz) von Varietäten (cf. Kerswill/Williams 2002). Neben der Varietätenkonvergenz und -divergenz ist auch long term dialect accommodation (also die Anpassung der Sprechweise einzelner Personengruppen an andere, z.B. aufgrund von Migration innerhalb einer Sprachgemeinschaft oder von einem englischsprachigen Land in ein anderes, cf. Siegel 2010; Auer/Barden/Großkopf 1998; Chambers 1992 u. a.) unter Verwendung des Salienzbegriffs untersucht worden. Die empirisch zu beantwortende Frage ist, ob die Salienz eines Merkmals sein Verhalten in solchen Prozessen der Konvergenz und Akkommodation beeinflusst. Konkret stellt sich bei den meisten Untersuchungen die Frage: wird ein auffälliges Merkmal der eigenen Sprache leichter aufgegeben und ein auffälliges Merkmal der Zielvarietät leichter angenommen?

Allerdings muss bei einem Wandel $\mathrm{A} \rightarrow \mathrm{B}$ die Salienz der abgelegten Merkmalausprägung A und die der angenommene Merkmalsausprägung nicht identisch sein. Konvergiert eine Sprechergruppe mit einer anderen, muss einerseits die Salienz des fraglichen Merkmals in der eigenen Sprache in dieser Sprechergruppe bestimmt werden (um Vorhersagen darüber zu treffen, wie schnell diese Merkmale abgelegt werden), andererseits ist es auch notwendig, die Salienz des akkommodierten Merkmals in der Sprache der Bezugsgruppe zu messen, der sich die konvergierenden Sprecher anpassen), um Vorhersagen darüber machen zu können, wie schnell dieses Merkmal angenommen wird.

Eine zweite wichtige Unterscheidung ist die zwischen Selbst- und Fremdwahrnehmung. Für den Dialektabbau zugunsten des Standards ist oft die Außenwahrnehmung primär. Das fragliche Merkmal, das von einer Sprechergruppe verwendet wird, sticht also zunächst für die sprachliche Umgebung heraus. In der Sprechergruppe selbst muss es deswegen noch nicht salient sein. So sind manche grammatische Regionalismen zwar für den Rest der Sprachgemeinschaft sehr auffällig, werden von den Sprechern selbst aber kaum bemerkt (etwa die nordwestdeutsche Verwendung des Verbs anfangen mit dem sein-Auxiliar: er ist angefangen, oder die bair. Serialisierung der Hilfsverben im Nebensatz : ...dass sie die Pflanzen im Garten vertrocknen lassen hat). Erst wenn die Fremdwahrnehmung zur Selbstwahrnehmung wird, wird sie für Wandel bzw. Akkommodation relevant.

Das hier vorgeschlagene Modell ist hierarchisch organisiert und geht davon aus, dass das physiologische Ursachen für Salienz den kognitiven und diese den sozialen nachgeordnet sind. Mit anderen Worten: Hohe physiologische Salienz eines Merkmals macht es zwar wahrscheinlicher, dass das Merkmal auch auf dem Hintergrund des kognitiven Bedingungsgefüges salient ist, genauso wie hohe kognitive Salienz es wahrscheinlicher macht, dass das Merkmal auch sozial auffällt. Die Beziehung ist jedoch nicht zwingend, denn kognitive Salienzbedingungen können auch bei geringer physiologischer Salienz ein Merkmal hervorheben, und soziolinguistische Salienzbedingungen können physiologisch und kognitiv wenig saliente Merkmale auffällig machen. Das hierarchische Modell macht überdies die 
Voraussage, dass physiologische Faktoren allein nur wenig zur Salienz eines Merkmals beitragen, kognitive Faktoren allein oder in Verbindung mit physiologischen etwas mehr; soziale Faktoren leisten jedoch deutlich den höchsten Beitrag zur Salienz. Der Grund dafür ist, dass Faktoren der unteren Ebene durch das "Filter" der höheren gehen, soweit solche Faktoren der höheren Ebene überhaupt relevant sind. Nur wenn das nicht der Fall ist, können sie überhaupt wirksam werden, anderenfalls haben sie höchstens verstärkende Funktion:

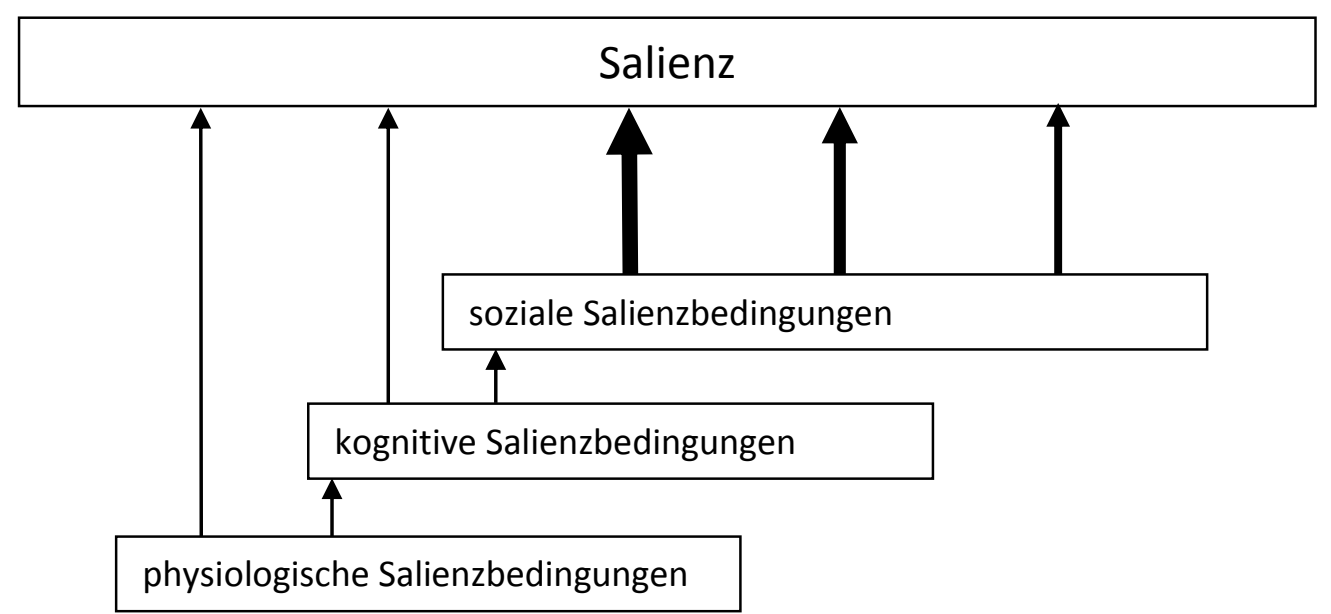

Salienz tritt nun als ein Faktor in ein Modell von sprachlicher Konvergenz und Akkommodation ein. Gemäß dem Modell werden Konvergenz und Akkommodation am meisten von sozialen Salienzbedingungen beeinflusst, die Bedeutung physiologischer und kognitiver Faktoren ist nur gering. (Sie sind allerdings nicht ganz irrelevant, wie die Beobachtung zeigt, dass Sprachwandel oft in den physiologisch salientesten Umgebungen beginnt.) Aber ohne weitere Faktoren kann das Modell Sprachwandel und Akkommodation nicht erklären. Das ist zuallererst die Polarität der sozial-affektiven Bewertung, die ein soziolinguistisches Merkmal erfährt. Es ist offensichtlich, dass ein positiv bewertetes Merkmal der eigenen Gruppe nicht (schnell) abgebaut wird, ebenso wie es naheliegend ist, dass ein positiv bewertetes Merkmal der Bezugsgruppe, zu der hin die Konvergenz erfolgt, früh erworben wird. Ebenso ist es klar, dass ein negativ bewertetes Merkmal der eigenen Gruppe leichter aufgegeben wird, während negativ bewertete Merkmale der Bezugsgruppe nicht übernommen werden.

Nicht nur aus diesem Grund ist Salienz allein kein guter Prädiktor für Wandel. Es gibt andere Faktoren, die ebenfalls einen Einfluss haben, wie etwa die Leichtigkeit, mit der ein Merkmal erworben und in die eigene Sprechweise integriert werden kann. Zum Beispiel sind lautliche Merkmale, die an lexikalische Klassen gebunden sind, relativ schwer zu erwerben, weil sie von Wort zu Wort gelernt werden müssen. Auch bei hoher Salienz und positiver sozialer Bewertung scheitert daher ihr Erwerb nicht selten (cf. Payne 1980). Manche Merkmale sind also intrinsisch sehr schwer abzulegen - so erklärt sich vielleicht, dass das saliente und negativ konnotierte Phänomen der $c h$-Koronalisierung (sowohl in seiner dialektalen als auch in seiner ethnolektalen Variante) dennoch von manchen Sprechern kaum vermieden werden kann.

\section{Abschließende Bemerkungen}

Die Diskussion über Salienz in der deutschsprachigen und teils auch internationalen soziolinguistischen Literatur ist immer wieder von dem Versuch geprägt worden, für das offenbar 'subjektive' (weil wahrnehmungsbasierte) Phänomen der Auffälligkeit bestimmter Merkmale 'objektive' Erklärungen zu finden, die in der Art des untersuchten Merkmals selbst liegen. Ich glaube, dass wir uns von dieser Idee befreien sollten. Salienz ist ein Phänomen der Wahrnehmung, und gerade als Prädiktor für Sprachwandel und Akkommodation zudem ein Phänomen der sozialen Bewertung. Saliente Merkmale, die im Sprachwandel eine Rolle 
spielen, sind solche, die als 'Figuren' auf dem Hintergrund eines sprachideologischen 'Grunds' herausstechen. Es ist daher von vornherein vergeblich, sie auf rein kognitive Faktoren reduzieren $\mathrm{zu}$ wollen. Es erscheint sogar sinnvoll - wie in diesem Beitrag argumentiert anzunehmen, dass soziolinguistische Salienz ein Produkt (nicht eine Folge) sozialer Stereotypisierungen und anderer interaktiver Prozesse ist, durch die bestimmte sprachliche Formen Teil von Stilen und Registern werden (Agha's enregisterment). Wenn man dies akzeptiert, ist es letztendlich vielleicht besser, den Begriff der Salienz ganz zugunsten soziolinguistischer Theorien zur Bewertung sprachlicher Merkmale aufzugeben; Barbara Johnstone's Re-Interpretation der Silverstein'schen "indexikalischen Ordnung" (2003) wäre dafür ein guter Kandidat (cf. z.B. Johnstone et al. 2006).

\section{Literatur}

Anders, Christina A./Hundt, Markus/Lasch, Alexander (eds.) (2012): Perceptual Dialectology. Neue Wege der Dialektologie. Berlin: de Gruyter.

Agha, Asif (2003): "The Social Life of Cultural Value". Language \& Communication 23: 231-273.

Agha, Asif (2007): Language and Social Relations. Cambridge: Cambridge University Press.

Auer, Peter (1998): "Hamburger Phonologie. Eine variationslinguistische Skizze zur Stadtsprache der Hansestadt heute". ZDL LXV 2: 179-197.

Auer, Peter (2001): "Schirmunskis Unterscheidung zwischen primären und sekundären Dialektmerkmalen und ihre Bedeutung für die heutige Sozio-Dialektologie". In: Pankratowa, Svetlana M. (ed.): Nemetskaja Filologjja $i$ Sankt-Peterburgskom gosudarstwennom universitete. St. Petersburg, Universitätsverlag: 198-214.

Auer, Peter (2013): "Sociolinguistic Change, Indexical Fields, and the Longue Duree: Examples from the Urban Sociolinguistics of German". In: Anderson, Stephen R./Moeschler, Jacques/Reboul, Fabienne (eds.): L'interface langage-cognition/The Language-Cognition Interface. Actes du 19e Congrès Internaitonal des Linguistes, Genève, 22-27 juillet 2013. Genf/Paris, Droz: 201-232.

Auer, Peter/Barden, Birgit/Großkopf, Beate (1998): "Saliency in Long-term Dialect Accommodation". Journal of Sociolinguistics 2/2: 163-187.

Birkner, Karin/Gilles, Peter (2008): "Dialektstilisierung im Reality-Fernsehen". In: Christen, Helen/Ziegler, Evelyn (eds.): Sprechen, Schreiben, hören. Zur Produktion und Perzeption von Dialekt und Standardsprache zu Beginn des 21. Jahrhunderts. Wien, Praesens: 101-131.

Chambers, Jack (1992): "Dialect acquisition". Language 68: 673-705.

Eckert, Penelope (2004): "Variation and the Indexical Field". Journal of Sociolinguistics 12: 453-476.

Ellis, Nick C. (i. Dr.): "Afterword: Synthesizing frequency and associations within and across modalities and disciplines". In: Behrens, Heike/ Pfänder, Stefan (eds.): Frequency Effects in Language. Berlin: de Gruyter.

Elmentaler, Michael/Gessinger, Joachim/Wirrer, Jan (2010): "Qualitative und quantitative Verfahren in der Ethnodialektologie am Beispiel von Salienz". In: Anders, Christina A./Hundt, Markus/Lasch, Alexander (eds.): Perceptual Dialectology. Neue Wege der Dialektologie. Berlin/New York, de Gruyter: 111-149.

http://1.bp.blogspot.com/_0msJlvGVVi8/TOrinlLivRI/AAAAAAAAADY/vsEYPaJnNfM/s1 600/comic_doener.jpg, letzter Zugriff am 17.05.2014.

Johnstone, Barbara/Andrus, Jennifer/Danielson, Andrew E. (2006): "Mobility, Indexicality, and the Enregisterment of 'Pittsburghese'". Journal of English Linguistics 34/2: 77-104.

Kerswill, Paul/Williams, Ann (2002): "'Salience' as an Explanatory Factor in Language Change. Evidence from Dialect Levelling in Urban England". In: Jones, Mari C./Esch, Edith 
(eds.): Language Change. The Interplay of Internal, External and Extra-Linguistic Factors. Berlin/ New York, de Gruyter: 81-110.

Labov, William (1972): Sociolingustic Patterns. Philadelphia: University of Pennsylvania Press.

Lenz, Alexandra (2010): "Zum Salienzbegriff und zum Nachweis salienter Merkmale". In: Anders, Christina A./Hundt, Markus/Lasch, Alexander (eds.): Perceptual Dialectology. Neue Wege der Dialektologie. Berlin, de Gruyter: 89-110.

Payne, Arvilla C. (1980): "Factors Controlling the Acquisition of the Philadelphia Dialect by Out-of-State Children". In: Labov, William (ed.): Locating Language in Time and Space. New York, Academic Press: 142-178.

Rács, Peter (2013): Salience in Sociolinguistics. A Quantitative Approach. Berlin/Boston: de Gruyter.

Schirmunski, Viktor M. (1928/1929): "Die schwäbischen Mundarten in Transkaukasien und Südukraine". Teuthonista 5: 38-60, 157-171.

Schirmunski, Viktor M. (1930): "Sprachgeschichte und Siedelungsmundarten". GermanischRomanische Monatsschrift 18: 113-122 und 171-188.

Siegel, Jeff (2010): Second Dialect Acquisition. Cambridge: Cambridge University Press.

Silverstein, Michael (1981): The Limits of Awareness. Austin, Texas: Southwest Educational Development Laboratory. (= Sociolinguistic Working Paper 84).

Silverstein, Michael (2003): "Indexical Order and the Dialectics of Sociolinguistic Life". Language \& Communication 23/3: 193-229.

Stöckle, Philipp (2013): Subjektive Dialekträume im alemannischen Dreiländereck. Unveröffentl. Diss. Universität Freiburg: Germanistische Linguistik.

Trost, Pavel (1968): "Primäre und sekundäre Dialektmerkmale". In: Schmitt, Ludwig E. (ed.): Verhandlungen des 2. Internationalen Dialektologenkongresses. Marburg/Lahn, 5.10.9.1965. Band 2. Wiesbaden, Franz Steiner: 823-826. (=Zeitschrift für Mundartforschung 4).

Trudgill, Peter (1986): Dialects in Contact. Oxford: Blackwell. 